\title{
El focalizador aspectual guaraní hína en español paraguayo (jopara): significado, sintaxis y pragmática
}

\section{Introducción}

La situación de contacto entre las dos lenguas oficiales del Paraguay, el español y el guaraní, ha originado la emergencia de un continuum de hablas “jopara” ('mezcla' en guaraní), que se caracteriza por la alternancia de unidades procedentes de cada una de las dos lenguas. Entre estas alternancias, algunas son muy productivas, como es el caso del empleo, en la variedad más hispanizada de este continuum, de la forma de origen guaraní hína ${ }^{1}$ :

(1) Héctor Riveros apareció ayer con un raro look. El conductor de "Bien temprano" anda hína con el cabello largo que le molesta un poco a la hora de la conducción y, por eso, desde ayer salió al aire usando una vincha. ¿Qué tal le queda? (Crónica, 26/2/2015, "El raro look de Héctor Riveros”)

(2) De todos los personajes que eligieron para doblar, no hay uno favorito; "todos son especiales", porque eligen las series y los dibujos que les gustan. Orgullosos nos muestran sus trabajos. Comenzamos por el Chavo del Ocho, pasamos por Homero Simpson. "No es textual, como te digo; nosotros decimos cualquier cosa hína, así que tenés que atender”. Y bajo un insólito anuncio en los créditos de "Dirección hidráulica", nos muestran la serie del Chavo... Pasen y vean. (ABC Color, 6/10/2013, "Doblado al guaraní por tres roseños")

Como lo veremos más adelante, los estudiosos que se han interesado en la variante del español hablado en Paraguay han analizado este fenómeno como el préstamo morfológico del morfema de aspecto progresivo del guaraní (-ína) conjugado en $3^{\mathrm{a}}$ persona ( $h$-) -> hína. Ante esto, surgen entonces las siguientes interrogantes: i) ¿De dónde procede la forma guaraní?, ii) ¿Cuál es su funcionamiento

1 Para las palabras de origen guaraní, jopara [ḋopa'ra] y hína ['hina], conservamos aquí las grafías del guaraní normativo.

Élodie Blestel, Université Sorbonne Nouvelle, Rennes, France

Ә Open Access. (C 2019 Élodie Blestel, published by De Gruyter. (cc) BY-NC-ND This work is licensed under a Creative Commons Attribution-NonCommercial-NoDerivatives 4.0 International License.

https://doi.org/10.1515/9783110605679-009 
semántico y sintáctico en español? iii) ¿Cómo podemos analizar y explicar sus usos pragmáticos?

Para tratar de entender mejor este fenómeno lingüístico, trataremos primero de descubrir de dónde procede la forma -hína $[h-+$ ína $]$ en diacronía. Luego, intentaremos hacer una síntesis de la literatura acerca de $h$-ína $-\mathrm{y}$ las demás formas del paradigma conjugado- en guaraní y también en castellano paraguayo, para cotejarla con lo que podemos observar en un corpus de castellano (jopara) paraguayo en diarios de los últimos cinco años. Estas observaciones nos conducirán a formular la hipótesis siguiente: si queremos conservar un análisis de esta forma en términos de aspecto, habrá que entenderlo en un sentido amplio, ya que la aspectualización con hína no incide necesariamente en la construcción del evento en sí, sino más bien en la operación de co-construcci ón de la significación con el alocutario. Dicho de otra manera, lo que se aspectualiza con hína no es el desarrollo del evento sino el proceso selectivo de acceso, tratamiento y paulatina co-construcción de la significación entre el hablante y el alocutario.

Si lo interpretamos así $-\mathrm{y}$ es lo que trataremos de mostrar con este trabajo-, podemos entender por qué, en la mayoría de los casos, el morfema hína funciona como un focalizador atencional gramaticalizado, en el sentido en que obliga al alocutario a distinguir una parte cumplida, presupuesta, de otra parte por cumplir (y por ende, por construir), lo que abre la brecha para usos más bien modales y pragmáticos. En efecto, veremos que el análisis de este morfema en términos de focalización atencional es lo que nos permite explicar que el papel pragmático de hína pueda llegar a ser el de un morfema con el cual el hablante establece un contraste entre su propuesta lingüística y el punto de vista alocutivo anticipado en tres contextos -que no se excluyen mutuamente-: cuando el morfema aparece junto con un predicado estativo, cuando aparece junto con verbos cuyo morfema aspectual o temporal impide la relevancia del predicado en el momento de la enunciación (ver Tonhauser 2006, 275 e infra) y cuando su posición sintáctica sugiere un alcance que excede al del predicado, lo que le permite funcionar como marcador extraproposicional.

\section{La forma guaraní $h$-ína}

\subsection{H-ína en diacronía: posible origen y evolución}

Para poder entender esta forma en guaraní, empezamos primero por buscar su recorrido etimológico. Para eso, sacamos provecho de la existencia en línea de 
la nueva base de datos LANGAS (Lenguas Generales de América del Sur), ${ }^{2}$ la cual reúne un abanico de textos de toda índole (metalingüística, administrativa, religiosa) provenientes de los siglos XVI, XVII y XVIII. En lo que atañe al guaraní, la base contiene, en su fase actual, un corpus de 78 textos redactados entre 1630 y 1813. Ahora bien, si nos atenemos a la cantidad de los textos presentes en la base, sorprende que no aparezcan sino dos ocurrencias de hína, de la mano de un solo escritor, el Arzobispo de la Plata, en una carta redactada en 1787 a la atención de los indios Chiriguanos:

(3) 10. Cobaeco Jesu-Christo herabae, Tupa hae Abaete, ymombohapiha, arap̌pe omano rire oicobe yeb̆, oiupi Ybagape hecorupi, oguapı hina Tupa Tuba

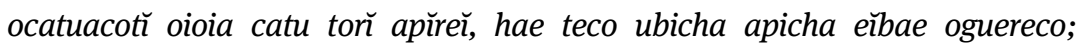
haèa egui ounune opondandubo mà oicobebae, hae omanobae cuèpe heco cuerarehe. (Carta del Arzobispo de la Plata a los Chriguanos, 1787. Fuente: LANGAS 2017). ${ }^{3}$

(4) 108. ñande Rey Carlos III. recou bicha, hae iporoporiahuberecohà oiapiç̌̆nune ombuata eı̌bo mbae amo iechupe cuera oiapo harami hina pendě̌p̆rupi oibae egui Taba p̌ahu mirimiri rupi, cona Salinas, Acero Pilipili, Aimiri, Tacuru, hae Masahabe. (Carta del Arzobispo de la Plata a los Chriguanos, 1787. Fuente: LANGAS 2017). ${ }^{4}$

2 Este proyecto, localizado en el Centro de Investigación del CREDA (IHEAL, Universidad Sorbonne Nouvelle) y financiado por la Agencia Nacional de Investigación francesa, reúne a investigadores - historiadores, antropólogos, filólogos y lingüistas - que se dedican al estudio de los documentos escritos que aparecieron en las épocas colonial y república temprana en lo que denominamos las "lenguas generales de Sudamérica", es decir, las principales lenguas vehiculares indígenas de uso extendido en esa zona (tupí, guaraní, quechua, aimara) que sirvieron de vehículo de comunicación entre hablantes de diversos idiomas amerindios y los europeos, lo que desembocó en la creación de nuevos espacios económicos y administrativos, así como en la evangelización de los indígenas.

3 “10. Este es el que se llama Jesu-Christo, Dios, y hombre verdadero, quien resucitando al tercer día, después de su muerte, y subiendo a los Cielos por su propia virtud, está allí sentado a la diestra de Dios Padre, es decir, con igual gloria, y Magestad, que él, desde donde vendrá a juzgar a los vivos, y a los muertos” (Traducción original).

4 “108. [D]onde nuestro Grande, y piadoso Rey, Carlos III. los recibirá, y mantendrá con la piedad, y magnificencia, que lo está haciendo en las Reducciones vecinas a vuestra Nación, como son las de Salinas, la de Acero, la de Pilipili, la de Aymiri, la de Tacurú, y la de Masahave” (Traducción original). 
También encontramos 3 ocurrencias de otra forma con morfema de 3 a persona, oina, fechadas en 1770 y una con la forma de 1 a persona (aína) con fecha 1810, reproducida en (5):

(5) 16. hae eupe bae rehe hape aiporu aina ñeê porâ hae teco porâ. (Carta de Manuel Belgrano a Don Pablo Thompson, 1810. Fuente: LANGAS 2017). ${ }^{5}$

Comprobamos que estas ocurrencias son muy pocas y relativamente tardías (finales del siglo 18). De la misma manera, si consultamos las obras de referencia para el guaraní colonial, a saber, El Tesoro de la lengua guaraní y el Arte de la lengua guaraní, publicados en Madrid en 1639 y 1640 por el padre jesuita Antonio Ruiz de Montoya, y el mismo Arte de la lengua guaraní anotado, por el padre Restivo y publicado en 1724, comprobamos que estas unidades no se mencionan con esta forma. No aparece ninguna ocurrencia de hína, ni en los diccionarios, ni en las gramáticas. Lo que sí aparece en cambio, es el verbo - $h \hat{\imath}$ 'estar', junto con el morfema -namo:

(6) Tupãópe oñembo'e hĩnamo,

'Estando rezando en la Iglesia' (Restivo 1724)

El morfema -namo no viene glosado como tal en estas obras de referencia pero, como solamente aparece en contextos nasales -es decir, inmediatamente después de una vocal nasal-, deducimos que se trata del alomorfo del morfema -ramo que funcionaba -y sigue funcionando hoy en día -como morfema de subordinación circunstancial, ya que permite construir oraciones subordinadas que circunscriben el evento o la situación de la oración principal a circunstancias precisas, por lo que se puede traducir, según los textos por 'cuando', 'si', 'en calidad de', o simplemente por un gerundio en español.

En el ejemplo facilitado por Restivo, se trata pues de una "construcción serial” en guaraní; a saber, aquella en la cual se acumulan varios verbos independientes sin ningún elemento de relación entre ellos, en este caso:

(7) o-ñembo'e / h-ĩ-namo

P3-rezar / P3-estar-SUBORD.

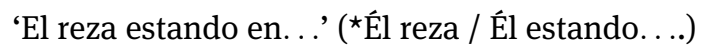

5 “16. [Y] es por esto que me valgo de los medios que dicta la prudencia, y la ley" (Traducción original). 
Parece ser, pues, que con la forma actual hína estamos ante la forma apocopada de la forma antigua hĩnamo, que podríamos glosar como 'estando', 'si está', 'cuando está', etc., según los contextos. Esta forma habría aparecido a finales del siglo 18, si nos atenemos a lo que encontramos en el corpus LANGAS.

\subsection{H-ína en la literatura gramatical y lingüística}

Hasta ahora, no hemos encontrado nada sobre los orígenes de la forma hína en la literatura sobre la lengua guaraní. Lo que sí subrayan todos los gramáticos es que en el guaraní normativo contemporáneo, el morfema -ína se asocia con "una especie de flexión para las distintas personas [...]” (Guasch 1996 [1956], 123); a saber, la serie de morfemas personales agentivos $a-/ r e-/ o-o^{-6} / \tilde{n} a-/ p e-$, de esta forma: a-ina/re-ína/o-ína/ñaina/peina.

Ahora, difiere según los autores la forma con la que describen el significado del morfema -ína, ya que puede verse como una marca de aspecto "progresivo" (Gregores y Suárez 1967, 144 y 155, Palacios 1999, 66), “continuativo” (Zarratea 2002, 88, Palacios 1999, 66), o "imperfectivo" (Krivoshein de Canese y Acosta Alcaraz 2007, 96). Puede tener consecuencias en cuanto a la "duración del evento" (Palacios 1999, 66) cuando se afirma su "realización continúa" (Zarratea 2002, 88), o que se trata de "una acción prolongada" (Krivoshein de Canese y Acosta Alcaraz 2007, 96). Sin embargo, algunos autores subrayan su función de comentario "[p]ara hacer resaltar la actuación o ejercicio del verbo" (Guash 1996 [1956], 123) e incluso "para resaltar el hecho de que algo está ocurriendo en el presente"7 (Gregores y Suárez 1967, 155), pudiendo -ína incluso aparecer en el comentario de oraciones ecuativas ${ }^{8}$ (ibid.). Por su parte, Dessaint

\footnotetext{
6 Zarratea $(2002,88)$ comenta al respecto de este morfema de $3^{\mathrm{a}}$ persona que "[e]n la $3^{\mathrm{a}}$ persona del singular y del plural la /o/ cambia por /h/ al repetirse" y Krivoshein de Canese y Acosta Alcaraz $(2007,96)$ que “[e]n la $3^{\mathrm{a}}$. persona se usa la inicial $h$ en vez del prefijo $o$ ”.

7 "They give progressive meaning to a verbal phrase and are used - when no other modifier indicates past action - to stress the fact that something is occurring in the present” (Todas las traducciones son nuestras).

8 "hina can also occur in the comment of an equational clause (cf. 16.1)". Estos autores definen las oraciones ecuativas como aquellas que consisten en un tópico y un comentario en una construcción predicativa atributiva (Gregores y Suárez 1967, 169 "consists of a topic and a comment in predicative attributive construction") pero no explicitan cómo se vinculan los dos tipos de empleo (empleo de hína con sentido progresivo vs empleo como comentario), y tampoco dan muestras de estos empleos específicos.
} 
afirma que -ína manifiesta la coincidencia del tiempo de enunciación con el tiempo de acción ${ }^{9}$ (Dessaint 1981, 178), lo cual viene corroborado por el análisis de Tonhauser (2006, 275):

En suma, hĩna es un marcador progresivo que es compatible con predicados dinámicos y estativos. Con los primeros se afirma la continuidad de la descripción de la eventualidad, mientras que afirma la relevancia inmediata o temporalidad del estado con el último tipo de predicado. ${ }^{10}$ (Tonhauser 2006, 275)

\subsection{Hína en el continuum jopara}

En cuanto a los escritos sobre la presencia de la forma en el español hablado en Paraguay, aparece mencionada la forma hína por primera vez en la obra de Usher de Herreros (1976), pero no como forma prestada sino como origen de un empleo más abundante de la perífrasis <estar + gerundio>. Según esta autora, la forma hína actúa como "modificador de la acción” en guaraní: "Si omba'apo puede significar trabaja o trabajó, el sufijo hína que actúa de 'modificador de la acción' descarta la duda de que 'Prudencio trabaja'” (Usher de Herreros 1976, 68).

Ahora, esta autora no menciona la presencia de hína en español paraguayo ya que, según ella, el guaraní-hablante encuentra en las formas perifrásticas del presente progresivo una forma equivalente al guaraní hína. Granda (1988, 167ss.), en cambio, es el primero en mencionar su presencia como préstamo en enunciados en español, pero al calificarla de "marcador aspectual continuativo" en enunciados en tercera persona, la analiza en términos de "duración de la acción”, al igual que en su último trabajo sobre el tema unos diez años más tarde:

El español paraguayo (en sus registros más cercanos al polo vernáculo) ha adoptado e incorporado, por lo tanto, a su estructura verbal el marcador morfológico de aspecto durativo existente en el guaraní local, hína, con el mismo valor que posee en la lengua prestataria [.. . ] (Granda 1999, 169).

En los estudios posteriores, la mayoría de los autores ha acatado el hecho de que la forma hína se ha incorporado en el español local y la acota como forma

\footnotetext{
9 "manifeste la coïncidence du temps d'énonciation et du temps d'action".

10 "In sum, hĩna is a progressive marker which is compatible with both dynamic and stative predicates. With the former it asserts the ongoingness of the eventuality description, whereas it asserts immediate relevance or temporaryness of the state with the latter type of predicate".
} 
de aspecto progresivo (Palacios 2005, 32, Gómez Rendón 2007, 536, Estigarribia 2015, 196, 211 y 212). Una propuesta que, sin embargo, llama la atención es la de Kallfell (2016). En su trabajo sobre la gramática del jopara, Kallfell estudia un corpus de variantes mucho más guaranizadas (no se calificarían de español) y observa que esta forma está desplazando las demás formas del paradigma conjugado, al utilizarse hína incluso con verbos conjugados en $1^{\mathrm{a}}$ o $2^{\mathrm{a}}$ persona. ${ }^{11}$ Por otra parte, propone analizarla como "partícula asertiva" e incluso como marcador evidencial:

En conclusión hay que decir que el jopara, sobre todo el hablado por la capa social menos formada de la ciudad y el hablado en el campo, tiende a generalizar hína como forma no conjugada para la expresión de la visión angular. ${ }^{12}$ Pero además el singular hína, al igual que el plural hikuái, que originalmente comportaban un significado aspectual, sobre todo en el ámbito urbano han cambiado parcialmente su función, transformándose en partículas asertivas. Con esto el jopara muestra una gramaticalidad autónoma en el campo de la evidencialidad y se ha alejado también aquí del guaraniete [i.e del guaraní "puro", sin rasgos de contacto con el español]. (Kallfell 2016, 126, énfasis nuestro)

El trabajo de Kallfell es interesante porque aporta datos nuevos sobre el uso de esta forma en hablas espontáneas y muestra cómo esta forma va adquiriendo cierta autonomía. Como hemos dicho en la introducción de este trabajo, queremos mostrar que el análisis en términos aspectuales no es necesariamente contradictorio con los efectos pragmáticos que podemos percibir en el continuum jopara, ya sea en las variantes más cercanas a la lengua española (las que vamos a describir aquí) o en las que se acercan más al polo opuesto, como lo son por ejemplo las estudiadas por Kallfell (2016). Veamos ahora lo que hemos podido observar en periódicos paraguayos editados en los últimos cinco años.

11 Este hecho ya aparece comentado también en Gregores y Suárez: "For the third person the form hína is used, but it cannot be properly considered as inflected for third person, because it also occurs with verbs inflected for any of the other persons" $(1967,144)$.

12 El autor retoma aquí el concepto coseriano de "visión parcializadora angular" (Coseriu 1976) por medio de la cual el hablante enfoca la acción verbal entre dos puntos de su desarrollo, desde un ángulo determinado, ya sea acentuando su totalidad o unicidad (visión globalizadora) o bien, partes de su transcurso (visión parcializadora). En este trabajo, Kallfell define este concepto de la siguiente manera: "La visión angular parcializadora pone el foco en un sector de la acción, que está entre un punto A previo o simultáneo en relación a un punto C prefijado y un punto B posterior o simultáneo” (Kallfell 2016, 116). 


\section{Hína en la prensa paraguaya actual: datos del corpus}

Presentaremos aquí unas muestras de enunciados recogidos en diarios paraguayos editados en línea en los cinco últimos años. Dada la cantidad de ejemplos, hemos aplicado algunas pruebas en pos de consolidar o descartar nuestra hipótesis.

\subsection{H-ína versus a-ína/re-ína/pe-ína, etc.}

Como primera prueba, queríamos comprobar la presencia de las formas del paradigma conjugado de -ína en la prensa paraguaya. La búsqueda no toma en cuenta la variante (español jopara $<\ldots>$ guaraní jopara) pero ya da resultados bastante significativos en cuanto a la preponderancia de la forma hína con respecto a las demás (Tabla 1):

Tabla 1: Presencia del paradigma conjugado de -ína en la prensa paraguaya actual (2013-2017).

\begin{tabular}{lll}
\hline & SINGULAR & PLURAL \\
\hline P1 & Aína: 45 & Ñaína: 2 \\
P2 & Reína: 0 & Peína: 3 \\
P3 & \multicolumn{2}{c}{ Hína: 189 / Oína: 0} \\
\hline
\end{tabular}

Hay que aclarar que la forma grafiada aína o aina es también una interjección en guaraní, lo cual puede explicar su mayor presencia en el corpus. De esta primera prueba podemos deducir que es sobre todo la forma hína la que se emplea en este corpus periodístico. Un análisis cualitativo permite comprobar que su uso es casi exclusivo en los enunciados mayoritariamente en español, aparte del uso de la interjección ;aina!/;aína! Estas primeras observaciones van en el sentido de Kallfell (2016), en la medida en que podemos suponer que la forma de tercera persona va desplazando las de las demás personas. ${ }^{13}$

13 Ahora bien, la mayor presencia de tercera persona también podría ser resultado de la preponderancia de discursos sobre terceras personas en este tipo de corpus. 
Ahora, para discutir las propuestas anteriores acerca de la interpretación de hína, ${ }^{14}$ necesitamos comprobar en qué medida funciona como morfema gramatical de aspecto progresivo o continuativo y cómo se combina con los morfemas de tiempo y de aspecto gramatical de los verbos españoles. Luego, observaremos cómo se combina con el aspecto léxico (Vendler 1967) de estos mismos verbos, como han sugerido otros autores - aunque tratándose de su empleo en guaraní-, en particular Gregores y Suárez $(1967,155)$ y Tonhauser (2006, 275).

\subsection{Hína: ¿morfema de aspecto progresivo/durativo?}

Muchos contextos discursivos apuntan hacia un análisis en términos de progresión/duración de la acción; por ejemplo, cuando aparece la forma junto con un presente de indicativo:

(8) Cachíque no da respiro a nadie. Ayer, en un partidazo bien calidá venció a Libertad por 1 a 0 y se ubicó al ladito de Cerro Porteño en la punta del torneo Apertura. Su mensaje es bien clarito, quiere y mucho voi [desde luego] el título del torneo actual, a sabiendas que también tiene hína sus compromisos por la Copa Libertadores de América (Crónica, 3/5/2015, “Guaraní ganó y calentó el Apertura”).

(9) Romerito reveló que anda hína de novio. "Estoy en pareja, tengo una compañera porque novia ya no se puede decir a mi edad. Ella es de Ciudad del Este, pero no voy a decir su nombre", dijo el expelotero que no quiso entrar más en detalles sobre su noviazgo (Crónica, 14/10/2015, "Romerito contó que tiene chica'i [novia]").

Este tipo de empleos tal vez constituya el origen del análisis de la forma hína como equivalente de la perífrasis española <estar + gerundio> (Usher de Herreros 1976, 68), pero es de notar que, en español paraguayo, también puede aparecer el morfema junto con la perífrasis española <estar/andar + gerundio>, siempre en postposición, pudiendo estar separado de la perífrasis por un adverbio, como es el caso de los ejemplos (10) y (12) ${ }^{15}$ :

14 Ver supra, Secciones 2.2. y 2.3.

15 Ver también infra, Sección 4.2.3. 
(10) “Me parece superbién la llegada de Chiqui al Olimpia. Es un buen técnico y puede aportar mucho al club, ya que estos últimos partidos no han podido ganar. Esperemos que le vaya bien" le bajó la patrona del "Tito" Torres, que anda jugando bien hína en los últimos partidos (Crónica, 17/3/ 2015, "Kalé Galaverna: "Mi club se ha convertido en basurero de Cerro Porteño”).

(11) Y hablando del zaguero liberteño, su futuro inmediato estaría en el fútbol de Catar. En la "Huerta” llegó la oferta y se está estudiando hína (Crónica, 5/6/2015, "No vamos a ir solo a participar").

(12) Cecilio Domínguez fue llamado ayer para reemplazar a "Pájaro" Benítez. El delantero está jugando purete hína (Crónica, 4/10/2016, “Llamó a Cecilio Domínguez").

Por otra parte, la observación de los contextos de hína muestra que no se trata solamente de empleos en tiempo presente. Los empleos con el imperfecto del indicativo, si bien son compatibles con una visión imperfectiva de la acción, descartan la posibilidad de analizar esta forma como "una coincidencia del tiempo de enunciación con el tiempo de acción” como lo proponía Dessaint (1981, 178), para el guaraní:

(13) El conductor de "Karu porã" iba hína en su camioneta SsangYong, tipo Korando, con chapa KAO 363 y cuando alcanzó la esquina de Mariscal López y Estados Unidos chocó contra un auto de la marca Kia, Piccanto, con chapa GAG 077, guiado por Adriana Sagia. El vehículo de la doña debido al impacto volcó por completo. (Crónica, 13/6/2015, "Famoso cocinero casi hizo puré a doña”)

(14) La "máquina" se descompuso ya que se le salió la cadena a metros de donde lo robaron y no pudieron continuar con su escape. Los vecinos del lugar estaban vicheando hína ra'e [resulta que los vecinos del lugar estaban espiando] el asalto y sin pensar dos veces los redujeron patada púpe [a patadas] y tongo aku porã por su cara [y golpes bien fuertes en la cara], por lo que se entregaron cual manso cordero en el matadero. (Crónica, 24/5/2017, "Levantaron una moto, oñembyai [descompusieron] y ligaron [fueron castigados] mal”)

También encontramos empleos en futuro de indicativo (15), e incluso -lo que es más problemático aún si es que queremos analizar hína como marca de 
aspecto gramatical, exclusivamente- ${ }^{16}$ encontramos usos de hína con verbos conjugados en tiempo pretérito de indicativo (16).

(15) Capeto “cafetero” llegará hoy hína (Crónica, 23/4/2017, “Capeto 'cafetero' llegará hoy hína”).

(16) Indert también tuvo su festichola hína (Crónica, 24/6/2017, "Indert también tuvo su festichola hína”).

Podríamos glosar este último ejemplo (16) como un equivalente de "estuvo teniendo su festichola", pero otros empleos de pretérito con "ya” impiden hacer semejante análisis:

(17) Ya vendieron más de 2.500 combos hína. Cerro Porteño ya vendió hasta ayer más de 2.500 combos de entradas para ver el partido contra el DIM el martes por la Sudamericana y Guaraní por la fecha 16 del Clausura (Crónica, 21/10/2016, “Dos chutes abren la venta 15”).

(18) Muitu [mucho] espléndor por tu cumple, ya te saludé hína en Vive. Pero igual vos no estás ni ahí (TVO news, 14/3/2017, "Clara Franco y su simpático saludo de cumple a Walter Evers").

\subsection{Hína: ¿morfema de "relevancia inmediata" con los predicados estativos?}

Por otra parte, como hemos señalado, algunos autores apuntan hacia un análisis en términos de la relación entre hína y el aspecto léxico del predicado: en aquellas construcciones con predicados estativos en las que no podemos conceptualizar etapas diferentes en el desarrollo de la acción, estos autores proponen interpretar hína en términos de "relevancia inmediata" (Tonhauser 2006, 275) o de “comentario” (Gregores y Suárez 1967, 169), lo cual aboga por una interpretación pragmática que parece alejarse de la lectura progresiva hasta aquí comentada. Estas dos propuestas -que fueron pensadas para la

16 Recordemos que esta lectura es la que propone la gran mayoría de los estudiosos que han estudiado el préstamo de hína en español paraguayo (Palacios 2005, 32, Gómez Rendón 2007, 536, Estigarribia 2015, 196, 211 y 212), con la excepción de Kallfell (2016, 126). Ver supra, Sección 2.3. 
lengua guarani ${ }^{-17}$ también se ven corroboradas por los empleos de hína junto con predicados estativos en español:

(19) Ser puntual no existe hína para el paraguayito. Si te dicen un horario para hacer algo mínimo 20 min antes tenés que estar no después ni justito a la hora. Cine a las 20:00, 19:30 ya aparecés vos con tu entrada en mano. (ABC Color, 5/7/2014, “Cómo ser un caballero en el siglo 21”).

(20) Óscar Romero parte de titular. El "melli” sabe hína de goles (Crónica, 6/9/2016, "Recontramachos trancan hoy").

(21) Y cuando las autoridades pierden la confianza de la gente, no es hína sencillo remontar. (Prensa cooperativa, 13/5/2016, "Ciego y sordomuda”).

Ahora bien, sostenemos que estos usos modales no se limitan a la presencia de predicados estativos como los que acabamos de exponer: si mantenemos el criterio de la combinación de hína con el aspecto léxico, también comprobamos que aparecen usos pragmáticos con predicados dinámicos asociados con aspecto gramatical perfectivo, como en el caso del ejemplo (22) a continuación:

(22) Todo un furor fueron las chipas que prepararon en la cabina de la aplastante número uno, no sobro anga ninguno. ¿Y vos pa [morfema interrrogativo] che [mi] socio ya hiciste tu chipa hina hoy? (Farra Play, 28/3/2018, “Chipo apo 'en el kiosco””)

Si hína fuera sensible al aspecto léxico podría entenderse que aparezca con aspecto gramatical perfectivo, en la medida en que el evento sea internamente durativo, pero aquí la presencia del adverbio "ya" impide mantener este análisis. Por otra parte, también aparece con predicados estativos asociados con aspecto gramatical perfectivo, aquí en (23):

(23) [E]sta carajeada fue para mi hina tocayo... parece que lo que le dije les pico demaciado... [sic] (Motores, 8/1/2018, "Relatos de lo que puede hacer un subaru”)

17 Ver supra, Sección 2.2. 


\subsection{Primer balance}

Con lo que hemos visto hasta ahora, podemos concluir que la forma hína, que proviene probablemente de la apócope de la combinación de la forma antigua del verbo -ĩ 'estar' conjugado en $3^{\mathrm{a}}$ persona ( $\left.h \tilde{i}\right)$ con el morfema de subordinación circunstancial nasal -namo (ver supra, Sección 2.1) se ha gramaticalizado hasta convertirse en una forma no flexional desplazando las demás personas del paradigma (Secciones 2.2, 2.3 y 3.1). Se emplea en español paraguayo con verbos estativos y dinámicos, conjugados en tiempos verbales de aspecto imperfectivo y perfectivo. Estos datos ponen en tela de juicio el análisis de la forma exclusivamente en términos de aspecto progresivo, durativo o continuativo (Sección 3.2), y abogan por otra perspectiva, más atenta a sus usos modales y pragmáticos, como lo sugirieron algunos autores para el guaraní (Tonhauser 2006, Gregores y Suárez 1967), y también Kallfell (2016) para el español (Sección 3.3). En lo que sigue, tendremos dos objetivos para ahondar en el conocimiento de este morfema. Primero, avanzaremos un intento de explicación a la aparente paradoja entre el análisis del morfema en términos de aspecto progresivo y su propensión a funcionar con usos modales -incluso con verbos dinámicos-, proponiendo un significado sincrónico unitario para esta forma gramaticalizada, a saber, el de un operador aspectual de focalización atencional. Segundo, explicaremos cómo este operador unitario puede dar lugar a estrategias distintas a las de aspecto progresivo y en qué contextos precisos, tratándose aquí de sus usos en español paraguayo (y no en guaraní).

\section{Hína como operador de focalización atencional en español paraguayo (jopara)}

\subsection{Un enfoque enactivo de hína como operador aspectual de focalización atencional}

Como hemos podido comprobar, la forma de origen guaraní hína aparece en guaraní en el siglo 18 y presenta rasgos de gramaticalización, ya que se ha convertido en una forma no flexional que ha desplazado las demás personas del paradigma. No disponemos de un corpus diacrónico de español paraguayo, pero observamos que hoy en día hína también funciona como forma no flexional, teniendo usos pragmáticos que imposibilitan un mero análisis en términos de progresión de la acción. Lo que interesa aquí es, pues, explicar de qué manera puede hína contribuir a la emergencia de la significación en la experiencia 
dialógica, en su funcionamiento en sincronía, por lo menos cuando aparece en español en Paraguay.

Para ello, adoptamos el paradigma de la enacción en ciencias cognitivas (Varela et al. 1974, Varela 1996, Stewart et al. 2013) el cual considera que la cognición es la coordinación (posiblemente intersubjetiva) de procesos encarnados que permiten el advenimiento conjunto del cuerpo propio y del entorno. ${ }^{18}$ Aplicado a las ciencias del lenguaje, este paradigma implica concebir el lenguaje humano como una serie de acciones, comportamientos y coordinaciones intersubjetivamente compartidos que contribuyen a la emergencia de la significación (Bottineau 2013). Esta última, por tanto, no está predeterminada, puesto que surge a través de las formas interactivas que la producen. En este sentido, nuestra concepción del acto de habla viene a ser una concepción fenomenológica del lenguaje, puesto que las categorías conceptuales y referenciales surgen con y por el acto del habla, por su naturaleza de manifestación encarnada de interacción - de enacción, en el sentido vareliano- con el entorno.

Una primera consecuencia de lo que acabamos de exponer es que consideramos que ninguna forma lingüística $-\mathrm{y}$ tampoco hína- describe el mundo referencial externo (como si fuera algo prefacilitado), como tampoco traduce algún material mental o cognitivo preexistente: las formas lingüísticas, entre las cuales está hína, forman parte de aquellos comportamientos que contribuyen en cambio a la emergencia de la significación, es decir que contribuyen a la experiencia dialógicamente concertada de la conceptualización mediante el acto de habla.

Otra consecuencia de este acercamiento teórico tiene que ver con nuestra forma de enfocar lo que entendemos por "aspecto". Éste no puede ser visto como una mera traducción de un referente pre-determinado: el aspecto no traduce lo que está pasando en el mundo extralingüístico sino que vectoriza, verbalización mediante, el acceso a su conceptualización. El hablante puede valerse de esta herramienta lingüística o no, para referirse a una acción que dura ... o no ... Al final, lo que importa es cómo el lenguaje permite conceptualizar una forma de manera concertada con el alocutario. En el caso de hína, retomaremos la imagen de Bottineau (2012), que habla de "efecto escáner" o "estroboscópico" para dar cuenta de la doble progresión que permite tener acceso a la representación del evento:

18 Ver también Varela et al. (1991, 9), que afirman que "cognition is not the representation of a pre-given world by a pre-given mind but is rather the enactment of a world and a mind on the basis of a history of the variety of actions that a being in the world performs". 
El aspecto cursivo [...] se refiere de facto a la relación interlocutiva con la construcción de una representación del evento, que se concibe en su globalidad y sin análisis particular [...], o en su realización, analítica y diferencialmente, y sobre la base de algún predeterminado cognitivo adquirido o compartido [...], con un "efecto escáner" o "estroboscópico" [...] propenso a enfatizar los contrastes: hay una fusión formal de la progresión objetiva del evento y la progresión psicológica (determinada intersubjetivamente en el contexto del acto de habla) de la aprehensión de su representación (Bottineau 2012, 99). ${ }^{19}$

En efecto, para entender mejor los usos pragmáticos que detallaremos más adelante, hay que mencionar lo que acarrea el distinguir, en la conceptualización aspectualizada de un evento, una parte cumplida de una parte por cumplir, porque esto no se aplica solamente a predicados dinámicos. Cuando hablamos de aspectualizar la conceptualización, esto se puede aplicar a la construcción de un evento dinámico pero también -y es lo que comprobamos con hína en nuestro corpus- puede aplicarse a la conceptualización de eventos estativos. Lo importante es que, en ambos casos, la aspectualización de la conceptualización obliga a focalizar la atención interpretativa sobre la distinción entre un <cumplido> $\mathrm{y}<$ por cumplir > mental, y sobre todo sobre la misma progresión atencional, ya que ésta resulta segmentada entre este cumplido mental (lo presupuesto y el conjunto de los saberes supuestamente compartidos) y un "por cumplir” mental que queda por construir, a saber, el dispositivo mediante el cual el hablante distingue la base de lo pre-determinado con una propuesta distinta desde el punto de vista alocutivo anticipado:

[...] el aspecto regula la sincronización coordinada de la co-determinación del objeto de representación (progresión del evento) y del sujeto que opera el acto de representación (detección del cambio, discriminación de diferencias, separación entre lo conocido y lo desconocido, transición de lo presupuesto a la inferencia, progresión del síntoma al diagnóstico y divergencia de una base de consenso hacia una propuesta polémica) (Bottineau 2012, 99)..$^{20}$

19 "l'aspect cursif [...] concerne de facto le rapport interlocutif à la construction d'une représentation de l'évènement, laquelle est appréhendée soit globalement et sans analyse particulière [...], soit dans son déroulement, analytiquement et différenciellement et sur la base d'un acquis ou accompli cognitif partagé [. . . ], avec un 'effet scanner' ou 'stroboscopique' [. . .] de nature à souligner les contrastes: il y a amalgame formel de la progression objective du procès et de la progression psychologique (déterminée intersubjectivement dans le cadre de l'acte de langage) de l'appréhension de sa représentation".

20 "[...] l'aspect régule la chronologie coordonnée de la co-détermination de l'objet de représentation (progression événementielle) et du sujet opérant l'acte de représentation (détection du changement, discrimination des différences, séparation du connu à l'inconnu, transition du présupposé à l'inférence, progression du symptôme au diagnostic, et divergence d'un socle consensuel vers une proposition polémique)". 
Al recurrir al morfema de aspecto hína, el hablante introduce una distinción entre una conceptualización dada, pre-determinada, una conceptualización que da por compartida, asimilada como ya existente, y otra por construir y cuya asimilación podría posiblemente ser objeto de desacuerdo con el alocutario. De ahí la posible lectura modal de hína, en particular con verbos estativos. Dicho de otra manera, lo que entendemos por aspectualización aquí no es necesariamente la de un evento en sí, sino que es la de la co-construcción misma (con el alocutario) de la idea de un evento o de un estado de cosas. Esto significa que no es como si la idea de un evento aspectualizado existiera primero y luego un morfema <aspecto progresivo> viniera a traducirla. Lo que proponemos es que es la conceptualización misma la que viene aspectualizada mediante hína. Para que se entienda mejor, podríamos representar el recorrido de conceptualización psicológica que vehiculiza el morfema aspectual hína con el siguiente esquema (Figura 1):

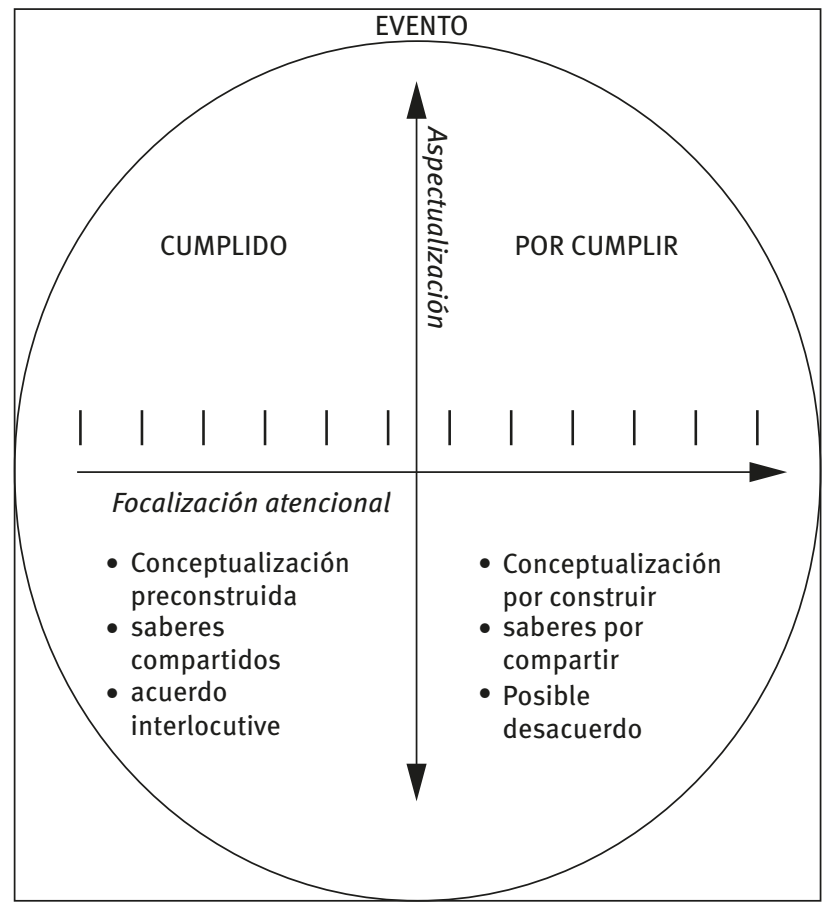

Figura 1: Hína: recorrido de conceptualización.

Lo que pretendemos mostrar con esta figura es cómo el hecho de aspectualizar obliga a distinguir dos partes (aquí representadas a la izquierda y a la derecha) 
que, para que puedan hacer surgir la representación de un evento -o de un estado- aspectualizado, parten primero de una distinción entre dos conceptualizaciones posibles (sea porque se concibe una parte cumplida y una parte por complir, sea porque se hace una distinción entre una conceptualización preconstruida y una por construir, una conceptualización ya compartida y una por compartir, etc.). Esto es lo que explicaría, a nuestro modo de ver, que coexistan varias interpretaciones posibles de hína según los contextos, y en particular, según el aspecto gramatical y léxico del verbo que va acompañando en contexto.

En (24), por ejemplo, sería difícil entender que, junto con el verbo ser, recurramos a un marcador de aspecto progresivo en sentido estricto de duración de la acción - por eso Tonhauser distingue dos usos en guaraní, es lo que hemos expuesto supra-. Pero si proponemos en cambio que hína funciona como un focalizador atencional que obliga al alocutario a recorrer el tratamiento y la paulatina co-construcción de la significación de 'es para facturar', entendemos que estos usos pragmáticos sean posibles: aquí, el uso de hína obliga el hablante a distinguir sus saberes preconstruidos de aquellos que quedan por construir. De esta manera, se le invita al alocutario a renunciar a sus eventuales expectativas ("Vanessa Insfrán se fue hasta Bolivia para facturar") tomando otro camino (> "Vanessa Insfrán se fue hasta Bolivia pero no para facturar”):

(24) Vanessa Insfrán viajó hasta Bolivia la semana pasada y no es hína para “facturar" (Crónica, 1/12/2016, "La paraguaya ndirindindin que encantó a los bolivianos”).

Aquí, hína marca morfológicamente el hecho de que se supone que el alocutario tal vez espere otra cosa: al aspectualizar la focalización atencional sobre la construcción del predicado, se invita al alocutario a recorrer el camino mental (el recorrido psicológico aspectualizado), para eventualmente cambiar de idea. Lo mismo pasa en (25), que podríamos glosar como 'este jueves ya es el juicio, a pesar de lo que uno se podría esperar':

(25) Cuentan que, incluso, una de las dos acusadas ni siquiera tenía abogado cuando fue notificada de que este jueves ya es hína el juicio (Crónica, 22/ 11/2016, "Pamela Vill: 'No soy Dios, no tengo nada que perdonarles”').

En efecto, en este artículo del periódico popular Crónica, el periodista cuenta que, según los rumores, los abogados de las acusadas no pensaban que los conflictos entre Pamela Vill y sus rivales fueran a desembocar en un juicio: "Según rumores, los abogados de las querelladas no tomaron muy en serio el asunto 
y no esperaron que la causa se eleve a juicio oral” (ibid.). La aspectualización de la focalización es un recurso para invitar a revisar una creencia tal vez errónea.

Los ejemplos de este tipo son numerosos y abogan por considerar el morfema hína, no en términos referenciales sino más bien en términos operacionales, es decir como pauta de orientación enunciativa. En (26) se comenta el caso de la extradición de Vilmar "Neneco" Acosta desde Brasil, para ser juzgado en el Paraguay por el asesinato de un periodista. El redactor de este ejemplo se queja de que los trámites entre los dos países posterguen el juicio cuando podía hacerse mucho antes. En esta oración, la presencia de hína después de "puede llevar meses" invita a que se focalice sobre la duración que tal vez no se esperaba ${ }^{21}$ :

(26) Lo que tendría que haberse hecho en un syky [un santiamén], ahora puede llevar meses hína (Crónica, 10/3/2015, "De esto se habla”).

De la misma manera, en (27), se comenta la implicancia de una modelo en el robo de un coche BMW falso que fue encontrado en su casa. El periodista confirma que, a pesar de estar fuera del caso por el momento, la posible culpabilidad de la señora seguirá siendo examinada "bajo la lupa de los investigadores" (ibid.):

(27) Por el momento, la modelo está fuera de la causa, pero seguirá siendo indagada hína (Motores, 5/10/2015, "El que mal anda, mal acaba”).

Este análisis del morfema hína en términos operacionales también permite entender por qué no podemos considerar hína como un mero equivalente de la perífrasis <estar + gerundio> como lo sugiriera Usher de Herreros (1976, 68). De hecho, aparece en los mismos contextos para aportar esta orientación de focalización atencional:

(28) Y hablando del zaguero liberteño, su futuro inmediato estaría en el fútbol de Catar. En la "Huerta” llegó la oferta y se está estudiando hína. Al Sadd SC sería el equipo interesado, el mismo que hace poquito nomás contrató al valécho del Barcelona, Xavi Hernández (Crónica, 5/6/2015, "No vamos a ir solo a participar").

21 En este caso, lo que acarrea la focalización atencional de hína es que lo que está por cumplir es la ideación, la emergencia de una significación tal vez nueva de la idea "puede llevar meses”. Dicho de otra manera, la focalización atencional implica la aspectualización de la ideación, y no de algo que no se haya cumplido todavía en en mundo extralingüístico, obviamente. 
(29) Cecilio Domínguez fue llamado ayer para reemplazar a "Pájaro" Benítez. El delantero está jugando purete hína (Crónica, 4/10/2016, "Llamó a Cecilio Dominguez").

Ahora bien, sostenemos que esta posibilidad no impide las posibles lecturas de hína como morfema de aspectualización progresiva, pero ésta igual se deduce del contexto de empleo, aquí junto con “ahora”, en (30):

(30) El portero santaniano Pablo Gavilán, cuyo pase pertenece al Ciclón, contó que en un trato con peloteros de la entidad azulgrana se acordó un premio para los del interior en caso que le tranquen al Decano el festejo el título, así lo hicieron y ahora se aguarda hína por la platita (Crónica, 8/12/2015, “¿Incentivo cerrista? “Esperamos la plata”, he’i Pablo Gavilán”).

En efecto, la interpretación de duración del evento no viene en el significado primario del morfema hína, ya que éste simplemente invita a distinguir dos fases en la interpretación psicológica del evento: aquí, entre lo cumplido y lo que queda por cumplir. Para decirlo de otra manera, aquí no se aspectualiza con hína el desarrollo objetivo del evento "aguardar" sino el proceso selectivo de acceso a la coconstrucción de la significación de "aguardar”. Pero esto no acarrea de forma automática un matiz de contraexpectativa: como se ve en (30), es posible interpretar hína como marca de progresión, es decir, como un morfema que remite a un evento que está cumpliéndose. Lo mismo puede decirse de los ejemplos (31) y (32):

(31) Hoy se pide hína el chute de "Neneco" del Brasil (Crónica, 30/3/2015, "Hoy se pide hína el chute de 'Neneco' del Brasil”).

(32) Nosotros no vemos hína youtube! (Motores, 14/1/2015, "Tema Oficial del Team Micro Machines")

Pero al no cumplirse en todos los casos esta posibilidad interpretativa, afirmamos que, cuando se emplea hína en español paraguayo, la propuesta según la cual se trata de un morfema de "relevancia inmediata" o "temporalidad del estado" (Tonhauser 2006, 275) sólo es válida en el caso de un predicado en tiempo presente. En cuanto a la propuesta de Kallfell (2016, 126), que ve en hína una partícula asertiva, proponemos precisarla explicando cómo de un significado de operador aspectual de focalización atencional, el morfema hína puede emplearse, en ciertos contextos, como marca de estrategia pragmática de focalización de contraexpectativa. Trataremos a continuación de circunscribir más detenidamente los contextos en los que es posible tal estrategia. 


\subsection{Usos pragmáticos de hína como foco de contraexpectativa}

Ahora que hemos definido el significado de hína como operador aspectual de focalización atencional, veamos cómo se explota en los usos pragmáticos que hemos observado en nuestro corpus. Como hemos sugerido, a veces la operación de focalización atencional recae sobre una propuesta informacional que se supone distinta con respecto al punto de vista alocutivo anticipado. Hemos identificado tres contextos -que no se excluyen mutuamente- que pueden contribuir a una interpretación de contraexpectativa: cuando el morfema aparece junto con un predicado estativo, cuando aparece junto con verbos cuyo tiempo impide la relevancia del predicado en relación con el momento de enunciación y cuando su posición sintáctica sugiere un alcance que excede al del predicado, lo que le permite funcionar como marcador extraproposicional (Blakemore 2002).

\subsubsection{Hína y predicados estativos}

Como hemos expuesto en la Sección 3.2, las construcciones en las cuales hína aparece junto con verbos estativos, invitan a otra interpretación que la de progresión, ya que no podemos conceptualizar etapas diferentes en el desarrollo de la acción. Es el caso de los verbos tener y querer del ejemplo (8) reproducido en (33) y del ejemplo (34):

(33) Cachíque no da respiro a nadie. Ayer, en un partidazo bien calidá venció a Libertad por 1 a 0 y se ubicó al ladito de Cerro Porteño en la punta del torneo Apertura. Su mensaje es bien clarito, quiere y mucho voi [desde luego] el título del torneo actual, a sabiendas que también tiene hína sus compromisos por la Copa Libertadores de América. (Crónica, 3/5/2015, "Guaraní ganó y calentó el Apertura")

(34) "Yo no busco de verdad pelearme con nadie, pero se pasa. Teniendo hijo ya, ni aún así deja de tirar mala onda. Pero bueno, opáma [se acabó], quiere hína buscar tema para que hable de algo interesante en su programa", le bajó Marilina bombardeando que Mily solo busca un tema de debate para el ciclo “En boca de lobos” (Crónica, 27/11/2017, “iLa Kchorrita y Marilina, en guerra por un jeans!”).

En el caso de (33), la presencia de la locución "a sabiendas" podría permitir una interpretación de hína como partícula asertiva como lo sugiere Kallfell 
(2016). ${ }^{22}$ Sin embargo, nos parece que, de acuerdo con sus usos en otros contextos, el periodista se vale de la posibilidad que ofrece hína de establecer una diferenciación entre el conjunto de los saberes preconstruidos y los que quedan por construir en cada uno de estos predicados estativos: al focalizar la atención sobre “tiene”, hína le permite al hablante anticipar un posible desacuerdo alocutivo, o por lo menos, que el alocutario no haya tomado en cuenta que "Cachíque" sí tiene sus compromisos con la Copa Libertadores. En cuanto al ejemplo (34), se retoma las palabras de la cumbiera Marilina quien comenta los motivos por los cuales otra mujer, llamada Mily, le busca problemas. Al explicar la actitud de su rival con el hecho de crear la polémica en su programa ("quiere hína buscar tema para que hable de algo interesante en su programa”), Marilina invita, con el empleo de hína, a revisar la situación con otra explicación.

\subsubsection{Hína y aspecto perfectivo o resultativo}

La combinación del morfema con un predicado cuyo aspecto es perfectivo o resultativo desencadena una interpretación semejante: en los ejemplos que siguen, la presencia de los pretéritos “compró" (35), "se hicieron” (36) y de la construcción pasiva con el verbo ser y el participio "fue sorprendido manipulando" (37) hacen que sea imposible interpretar los predicados en términos de progresión temporal. Al aparecer hína, invita a releer de forma retroactiva el predicado como algo que podría cuestionarse. Así, en el ejemplo (35), se cuenta que tres modelos famosas se han recibido como licenciadas. A pesar de su éxito, el periodista relata que algunos rumores dicen que en realidad son títulos mal habidos (“esa compró hína su título”), donde entendemos una vez más que lo que marca hína es una invitación a revisar lo que uno podría creer:

(35) Pero ello no implica que las mismas reciban solamente las congratulaciones por parte de la gente, pues en el famoso ñe'êmbeguépe [chismerio] saltan famosas frases acusatorias como "esa compró hína su título" o "alguien se lo regaló”, como queriendo empañar y desmeritar semejante logro (Crónica, 1/8/ 2015, “Modelos, acusadas de comprar título").

22 Por otra parte, nuestro objetivo es proponer un significado unitario para una misma forma, hína, pues postulamos la bi-univocidad del signo saussureano, es decir, a un significante corresponde un significado único y viceversa. De aceptar la propuesta de Kallfell (2016), no podríamos explicar cuál sería la relación entre aserción aquí y aspecto progresivo en otros contextos. 
En (36), hína aparece en el título mismo de un artículo de Crónica que da cuenta de la movilización de los camioneros contra el ingreso de vehículos bitrenes en Paraguay: a pesar de estar los camioneros esperando la orden de sus representantes de llegar masivamente a Asunción, se cuenta que también (cosa que tal vez no sea de esperar) se "hicieron sentir" en San Pedro:

(36) En San Pedro también se hicieron sentir hína (Crónica, 2/2/2018, "En San Pedro también se hicieron sentir hína”).

Otro caso interesante es el del ejemplo (37), donde se cuenta cómo los policías detuvieron a un individuo en flagrante delito de robo de automóvil. Ironizan los periodistas con el eufemismo "manipular" - que aparece entre comillas en el título del periódico- eufemismo que retoman en el cuerpo del artículo seguido de "hína":

(37) Un supuesto robacoches fue cachado por los volái de Luque en plena faena delictiva. El sujeto fue aprehendido en la vía pública por un supuesto hecho de tentativa de hurto de vehículo, cuando fue sorprendido manipulando hína los cables de un vehículo estacionado en las calles Teniente Rojas Silva y Azara, a eso de las 4:30 horas del sábado (Crónica, 4/3/2018, "Lo encontraron “manipulando" vehículo ajeno").

Como se ve aquí, la presencia de hína invita a releer de forma retroactiva el predicado como algo que podría cuestionarse, pues obviamente, la "manipulación" no es lo que parece, ya que se trata claramente para el ladrón de robar el coche. Notamos aquí que aparece en voz pasiva pero junto con una forma de gerundio. Ahora, nos parece que el empleo de hína no es redundante ya que ofrece aquí el efecto pragmático específico que hemos venido describiendo.

\subsubsection{Hína como marcador extraproposicional}

De forma más general, la variabilidad que podemos observar en cuanto a la posición sintáctica del morfema indica que éste funciona como marcador extraproposicional, lo que sugiere una ampliación de su alcance y cierta autonomía, rasgos que, según Llamas Saíz $(2010$, 197), son prototípicos de los marcadores discursivos. Puede así aparecer separado del verbo después de otro adverbio, aquí un adjetivo adverbializado en (38) o después del complemento del verbo (39): 
(38) Cruzando paletas con River Plate, Libertad empieza a recorrer el camino para la defensa de su título, obtenido en el Apertura de este año. La cuestión no se presenta tan fácil hína, pues todos los equipos se han reforzado y preparado de buena manera (Crónica, 8/7/2016, "River tanteará al Guma de entrada”).

(39) El corazón de Jonathan Santana tiene nueva dueña hína (Crónica, 6/4/2017, “¿Festejo de Santana para su novia?”).

También puede aparecer antepuesto al verbo como es el caso del ejemplo (40) a continuación:

(40) $Y$ ese hina es el "sindicalismo que persigue el gobierno neo-liberal de Cartes"; no importa que le metieron hasta a su perro en la función pública, lo que importa es su antiguedad, tapehona pembojahy pira, primera buena ación de Marito, esperemos que sigan por esta línea (La Nación, 25/2/2016, "Sindicalista defiende a exdirector de RRHH del Senado y amenaza con lista de periodistas planilleros”).

Esta libertad en las posiciones sintácticas permite incluirlo en la categoría de los marcadores discursivos del español paraguayo que se caracterizan por tener incidencia en el conjunto del enunciado. Así sería lícito compararlo con aquellos morfemas de origen guaraní (ko, ningo/nio, hina, ra'e, etc.) o hispánico - pero exclusivos del español paraguayo en este tipo de empleos- (luego/loo, lo que, había sido, etc.), que funcionan todos como morfemas de segunda posición en la organización informativo-estructural de los enunciados ${ }^{23}$ :

(41) Ese luego es amarillista, es un vendido, se dice que a ese se le paga... Es fácil hablar sin conocer, pues no estamos en esa situación (ABC Color, 11/10/2014, "Si querés ser periodista, dejá de pensar"). ${ }^{24}$

(42) SOBERBIO. Floyd Mayweather no quiere luego que lo comparen con ningún luchador (Crónica, 17/11/2017, “Lo trató de hormiga a Conor”).

23 Estudiamos la alternancia entre los morfemas ko, ningo y luego en Blestel (2017).

24 El marcador discursivo luego no significa 'después' en español paraguayo, sino que se ha convertido en un marcador epistémico que tal vez pueda traducirse por 'desde luego' en español peninsular. Ver Blestel (2017). 
(43) Rayitas?? ese ningo es el tambor de freno man [...] (Motores, 28/8/2015, "Cera en pasta para raspondes"). ${ }^{25}$

(44) Y le quiere ningo a su hija seguro papa jaja (Motores, 12/7/2016, "Debe ir Preso !!!”).

Cabe observar que muchas veces son morfemas que tienen en común una función deíctico-pragmática que consiste en señalar un elemento del mensaje, cualquiera que sea su estado informativo y cognitivo, añadiendo una información en cuanto a las coordenadas de la enunciación (epistemicidad, evidencialidad). Alternan morfemas de origen guaraní y de origen español, pero en este último caso de una forma innovadora con respecto a su uso en otras variantes. Parece, pues, que estamos ante un funcionamiento sintáctico bastante productivo en estas variantes de español. Con esta propuesta tratamos de entender cómo se explota la gramaticalización de hína para un tipo específico de estrategia pragmática, a saber, la focalización sobre una propuesta informacional que se supone distinta con respecto al punto de vista alocutivo anticipado, pero habría que estudiar el conjunto de marcadores que tienen un funcionamiento sintáctico y discursivo análogo en español paraguayo.

\section{Conclusiones}

La forma hína, probablemente procedente de la apócope de la forma antigua del verbo - $\tilde{\imath}$ 'estar' conjugado en $3^{\mathrm{a}}$ persona (hĩ) con el morfema de subordinación circunstancial nasal -namo se ha gramaticalizado hasta convertirse en una forma no flexional desplazando las demás personas del paradigma. En la actualidad, hemos propuesto que hína se emplea en español jopara paraguayo como operador de focalización atencional sobre la progresión del evento -o del estado de cosas-, lo que puede conllevar, en ciertos contextos, efectos pragmáticos de contraexpectativa. Esta propuesta de significado procedural unitario permite entender la aparente heterogeneidad de sus interpretaciones, ya que, según los contextos, se puede interpretar como marca de aspecto progresivo o como estrategia pragmática de foco de contraexpectiva. Sin embargo, si analizamos

25 Ningo corresponde a un morfema evidencial del guaraní que en español paraguayo también funciona como epistémico. 
hína en términos operacionales, es decir como pauta de orientación enunciativa, entendemos mejor cómo la marca de progresión aspectual no incide en la conceptualización de la duración objetiva del proceso, sino que atañe más bien a la progresión de la co-construcción de la significación con el alocutario. Esta propuesta también permite echar otra luz sobre los posibles vínculos entre las distintas interpretaciones de esta forma, y tal vez permita entender mejor los motivos por los cuales viene presentando estos usos pragmáticos.

Por otra parte, hemos identificado tres contextos - que no se excluyen mutuamente- en los que surgen estos usos de contraexpectativa: cuando el morfema aparece junto con un predicado estativo, cuando aparece junto con verbos cuyo morfema aspectual o temporal impide la relevancia del predicado en el momento de la enunciación, y cuando su posición sintáctica sugiere un alcance que excede al del predicado. Esto le permite funcionar como marcador extraproposicional, lo cual invita a insertar hína en el conjunto de marcadores que tienen un funcionamiento sintáctico y discursivo análogo en español paraguayo.

\section{Agradecimientos}

Esta investigación se ha desarrollado en el marco del proyecto "El español en contacto con otras lenguas II: variación y cambio lingüístico”, Ministerio de Economía y Competitividad/Fondo Europeo de Desarrollo Regional (FFI201567034-P, MINECO/FEDER). Agradecemos la atenta lectura y las sugerencias de Chrystelle Fortineau-Brémond (Université Rennes 2) y de Eric Beaumatin (Université Sorbonne Nouvelle).

\section{Corpus}

Noticias de Paraguay y el mundo en ABC Color [En línea], http://www.abc.com.py Crónica / El gran diario de la gente [En línea], http://www.cronica.com.py

Farra Play [En línea], http://www.farra.com.py

Hoy / En Paraguay y el Mundo [En línea], http://www.hoy.com.py

La Nación [En línea], http://www.lanacion.com.py

Motores [En línea], https://motores.com.py/

Prensa cooperativa [En línea], https://prensacooperativa.com

TVO news / Revista paraguaya de actualidad y farándula, http://www.tvo.com.py. 


\section{Base de datos}

LANGAS - Langues générales d'Amérique du Sud. Base de datos en línea. Paris: CNRS (Centre National de la Recherche Scienfique). www.langas.cnrs. fr. [24/7/2017].

\section{Referencias}

Blakemore, Diane. 2002. Relevance and Linguistic Meaning. The Semantics and Pragmatics of Discourse Markers. Cambridge: Cambridge University Press.

Blestel, Élodie. 2017. Ko, ningo, luego: An Enactive Approach to the Emergence of an Epistemic Subsystem in Jopara. Signifiances (Signifying) 1 (3).25-40.

Bottineau, Didier. 2013. Language and Enaction. En John Stewart, Olivier Gapenne y Ezequiel A. Di Paolo (eds.). Enaction. Toward a New Paradigm for Cognitive Science, 267-306. Cambridge (MA): MIT Press.

Bottineau, Didier. 2012. Les périphrases verbales “progressives” en anglais, espagnol, français et gallo: aspect, phénoménologie et genèse du sens. En Christine Bracquenier y Louis Begioni (eds.). L'aspect dans les langues naturelles. Approche comparative, 93-136. Rennes: Presses Universitaires de Rennes.

Coseriu, Eugenio. 1976. Das romanische Verbalsystem. Tübingen: Gunter Narr.

Dessaint, Michel. 1981. La langue guarani. Esquisse d'une typologie interne du guarani paraguayen contemporain. Tesis de doctorado. Université Paris IV-Sorbonne.

Estigarribia, Bruno. 2015. Guaraní-Spanish Jopara Mixing in a Paraguayan Novel. Does it Reflect a Third Language, a Language Variety, or True Codeswitching? Journal of Language Contact 8. 183-222.

Gómez Rendón, Jorge Arsenio. 2007. Grammatical borrowing in Paraguayan Guaraní. En Yaron Matras y Jeanette Sakel (eds.). Grammatical borrowing in Cross-Linguistic Perspective, 523-550. Berlin/New York: Mouton de Gruyter.

Gregores, Emma y Suárez, Jorge A. 1967. A Description of Colloquial Guaraní. The Hague/ Paris: Mouton de Gruyter.

Granda, Germán de. 1988. Sociedad, historia y lengua en el Paraguay. Bogotá: Publicaciones del Instituto Caro y Cuervo.

Granda, Germán de. 1999. La expresión del aspecto verbal durativo. Modalidades de transferencia lingüística en dos áreas del español de América. En Germán de Granda (ed.). Español y lenguas indoamericanas en Hispanoamérica. Estructuras, situaciones y transferencias, 161-173. Valladolid: Universidad de Valladolid/Secretariado de Publicaciones e Intercambio Científico.

Guasch, Antonio s.j. 1996 [1956]. El idioma guaraní: gramática y antología de prosa y verso. Asunción: CEPAG.

Kallfell, Guido. 2016. ¿Cómo hablan los paraguayos con dos lenguas? Gramática del jopara. Biblioteca Digital Curt Nimuendajú. En línea http://www.etnolinguistica.org/biblio: kallfell-2016-jopara[18/06/2018].

Krivoshein de Canese, Natalia y Corvalán, Graziella. 1987. El español del Paraguay en contacto con el guaraní. Asunción: Centro Paraguayo de Estudios Sociológicos. 
Krivoshein de Canese, Natalia y Feliciano Acosta Alcaraz. 2007 [2001]. Gramática guaraní. Asunción: ServiLibro.

Llamas Saíz, Carmen. 2010. Los marcadores del discurso y su sintaxis. En Óscar Loureda Lamas y Esperanza Acîn Villa (eds.). Los estudios sobre marcadores del discurso en español, hoy, 183-239. Madrid: Arco Libros.

Palacios Alcaine, Azucena. 1999. Introducción a la lengua y cultura guaraníes. Valencia: IVALCA.

Palacios Alcaine, Azucena. 2005. Lenguas en contacto en Paraguay: español y guaraní. En Carmen Ferrero Pino y Nilsa Lasso-von Lan (eds.). Variedades lingüísticas y lenguas en contacto en el mundo de habla hispana, 35-43. Bloomington: Books Library.

Restivo, Paulo s.j. .1724. Arte de la lengua guarani por el P. Antonio Ruiz de Montoya de la Compañía de Jesús con los escolios, anotaciones y apéndices del P. Paulo Restivo de la misma Compañía (Sacados de los papeles del P. Simon Bandini y de otros). Santa María La Mayor.

Ruiz de Montoya, Antonio s.j. 1639. Tesoro de la lengua guaraní. Compuesto por el Padre Antonio Ruiz, de la Compañía de lesvs. Madrid.

Ruiz de Montoya, Antonio s.j. 1640. Arte, y Bocabvlario de la lengua gvarani. Compuesto por el Padre Antonio Ruiz, de la Compañía de lesvs. Madrid.

Stewart, John, Olivier Gapenne y Ezequiel A. Di Paolo (eds.). 2013. Enaction. Toward a New Paradigm for Cognitive Science. Cambridge (MA): MIT Press.

Tonhauser, Judith. 2006. The temporal semantics of noun phrases. Evidence from guaraní. Tesis de doctorado. Stanford University.

Usher de Herreros, Beatriz. 1976. Castellano paraguayo: notas para una gramática contrastiva castellano-guaraní. Suplemento Antropológico 11(1-2). 29-123.

Varela, Francisco J. 1996. Invitation aux sciences cognitives. Paris: Seuil.

Varela, Francisco J., Humberto R. Maturana y Ricardo Uribe. 1974. Autopoiesis: The Organization of Living Systems, its Characterization and a Mode. Biosystems 5(4). 187-196.

Varela, Francisco J., Evan Thompson y Eleanor Rosch. 1991. The embodied mind: Cognitive science and human experience. Cambridge (MA): MIT Press.

Vendlern, Zeno. 1967. Linguistics in Philosophy. Ithaca: Cornell University Press.

Zarratea, Tadeo. 2002. Gramática elemental de la lengua guaraní. Asunción: Marben. 
